\title{
A CROSS-CULTURAL COMPARISON OF YOUNGER AND OLDER ADULTS' SIMULATED HIGHWAY DRIVING PERFORMANCE UNDER SINGLE AND DUAL TASK CONDITIONS
}

\author{
Bryan Reimer ${ }^{1}$, Bruce Mehler ${ }^{1}$, Joonwoo Son ${ }^{2}$, Anna E. Pohlmeyer ${ }^{1,3}$ \\ Jarrod Orszulak $^{1}$, Jonathon Long ${ }^{1}$, Joseph F. Coughlin ${ }^{1}$ \\ ${ }^{1}$ Massachusetts Institute of Technology AgeLab \\ \& New England University Transportation Center, USA \\ ${ }^{2}$ DGIST (Daegu Gyeongbuk Institute of Science \& Technology), South Korea \\ ${ }^{3}$ Center of Human-Machine-Systems, Berlin University of Technology, Germany \\ Email: reimer@mit.edu
}

\begin{abstract}
Summary: Driving is a complex psychomotor task that is often interrupted by secondary activities that divert attention away from the roadway. The risk of inattentive driving is known vary with age. The degree to which culture impacts these changes is less established. To study the impact of age and culture on drivers' capacity to manage dual task demands, we developed a parallel driving simulation in the US and Korea. We assessed the performance of 135 drivers divided into two age groups, younger (20-29) and older (60-69). Both age and cultural group differences in basic highway driving performance measures were observed. However, the relative impact of the dual task demands on driving performance was largely consistent across cultures.
\end{abstract}

\section{INTRODUCTION}

It has long been recognized that the ability to adequately manage and sustain attention is a critical requirement of safe driving and that inattention or distraction are significant factors in automobile crashes. Diversion of attention to secondary tasks is one of the largest contributors to inattentive driving and, consequently, to accidents (Stutts \& Hunter, 2003). Since driving an automobile is typically accompanied by secondary tasks (adjusting the radio or environmental controls, talking with a passenger or on a cell phone, interacting with a navigation device), an understanding of how drivers allocate attention and manage workload is important to inform the design of vehicles, devices, and distraction mitigation aids as well as driver education.

Heightened attentional demands can be expected to affect everyone. However, the impact on the older driver is of particular interest since an individual's capacity for managing multiple tasks simultaneously is known to decrease with age (McDowd, Vercruyssen, \& Birren, 1991; Rogers $\&$ Fisk, 2001). While absolute attentional capacity declines with age, the quality of judgment of typical drivers increases throughout the lifespan. In younger drivers, impaired judgment is often associated with excessive speed and alcohol use (Boyle, Dienstfrey, \& Sothoron, 1996) whereas older drivers have been observed to recognize possible limitations and regulate their behavior by limiting their exposure to situations in which they may be at higher risk (D'Ambrosio, Meyer, Coughlin, \& Mohyde, 2008). However, limiting driving is not always an option and other adjustments may be required to maintain safe driving conditions. When workload is high, there is evidence that some drivers engage in compensatory behaviors, such as moderating their driving speed, to manage workload (Harms, 1991). Late middle age drivers generally drive more slowly 
than younger drivers (Reimer, Mehler, Pohlmeyer, Coughlin, \& Dusek, 2006) and there is evidence that individuals in both age cohorts may decrease speed during some dual task conditions (Mehler, Reimer, Pohlmeyer, \& Coughlin, 2008).

The current study was designed to gather additional data comparing the performance of younger and older drivers during single task simulated highway driving and in response to the added demand of a secondary cognitive task. In addition to considering the effect of age, this collaborative project included participants at sites in the US and in South Korea. Although some cross cultural driving research has been carried out, much of this work has been actuarial or consisted of comparisons of self-reported driving behavior (Matthews, Tsuda, Xin, \& Ozeki, 1999). By conducting a driving simulation study using the same experimental protocol and equivalent instrumentation, it was our goal to explore the extent to which patterns of behavior generalize across samples as well as to illuminate possible cultural factors.

\section{METHODS}

\section{Participants}

Participants were required to: a) be between the ages of 20 and 29 or 60 and 69, b) drive on average more than two times per week, c) be in good health and free from a number of major health conditions such as cancer or uncontrolled high blood pressure, d) not take medications for depression or other psychiatric conditions, e) have a mini mental status score (Folstein, Folstein, $\&$ McHugh, 1975) greater than 25 and f) not have previously participated in a driving simulation study. US participants were required to sign an informed consent form.

\section{Simulator}

The US arm of the study was conducted in the MIT AgeLab fixed-base driving simulator "Miss Daisy". The Korean arm of the study was conducted in a simulator constructed to the same specifications. Commonalities included the same DLP projector, screen, model of personal computer and graphics card etc. but did not have the same vehicle form factor (see Figure 1).

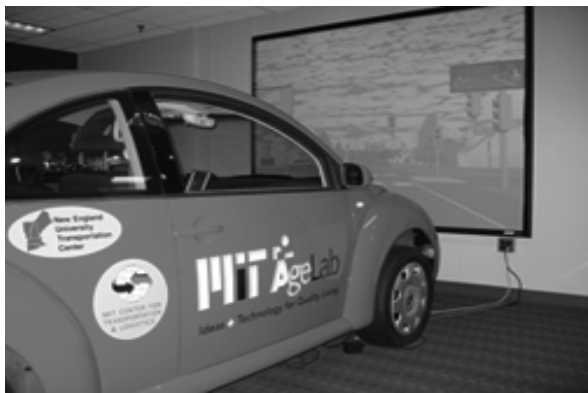

(a)

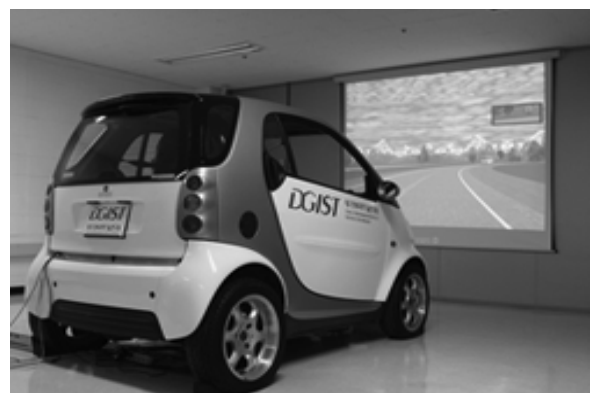

(b)

Figure 1. The MIT AgeLab driving simulator (a) and the DGIST simulator (b)

In both simulators, graphical updates to the virtual environment were computed using STISIM Drive $^{\mathrm{TM}}$ based upon inputs recorded from the OEM accelerator, brake and steering wheel. The 
virtual roadway was updated and data sampled at 20-30 Hz and displayed on an 8' x 8' screen at resolution of $1024 \times 768$. Parameters for STISIM Drive were adjusted in the US and Korea to account for differences in the vehicle form factors (eye height, rear view mirror placement etc.).

\section{Secondary Task}

An auditory cognitive task, n-back, was employed to induce changes in workload. The task, recommended by Zeitlin (1993) for inducing workload in transportation research, has been used in a number earlier studies in our laboratory (Mehler, Reimer, Coughlin, \& Dusek, 2009; Reimer, 2009). Unlike these earlier studies, participants were pre-trained on the task and were assessed independent of driving both before and after the simulation.

The version of the n-back task employed here consisted of a series of pre-recorded trials. Each trial lasted 30 seconds and was comprised of an aural presentation of a series of single digit numbers $(0-9)$ at an inter-stimulus interval of 2.25 seconds. Participants were asked to recall the " $\mathrm{n}$ "th" stimulus back in the sequence upon the presentation of the next digit. For additional background on the n-back task presentation see Mehler et al. (2009). The task was presented as a block of six trials. The first two trials employed a mild task demand (0-back), the second two trials a moderate demand (1-back), and the final two trials a high demand (2-back).

\section{Procedure}

After an introduction to the experiment, training on each level of the n-back task was provided. To facilitate learning, participants were given a written guide to follow along with the research assistant's verbal description and presentation of practice trials. Training on each level continued until satisfactory scores were obtained (no errors on a 0-back task, less than four errors on two consecutive trials of 1-back tasks, and less than five errors on two consecutive trials of 2-back tasks). To enhance the demands of driving the simulation, a financial incentive was used to encourage participants to maintain speed, obey the traffic laws, and devote attention to the secondary cognitive task (for details see Mehler et al., 2009; Reimer, D'Ambrosio, Coughlin, Kafrissen, \& Biederman, 2006).

Participants entered the simulator and drove a 1500m scenario designed to increase participants' familiarity with the simulator and promote habituation. They then stopped driving and completed a non-driving assessment of the n-back task (six trials) and a questionnaire. The primary simulation protocol followed. During this period, participants engaged in both an urban and a highway driving experience, each lasting approximately 15 minutes. The two segments were bracketed by transitional road conditions. No rest period was provided. The presentation order of the segments was counterbalanced such that half of the participants drove the urban setting first. (Results for the urban segment will be reported in a later paper.) Elements of the simulation are described in more detail in Reimer et al. (2006). During the second third of the urban and highway drives, the n-back task was again presented. To keep workload elevated over an equal driving distance for all participants regardless of their driving speed, additional trials of the 2back task were presented as needed. This procedure provided three equidistant periods of roadway in which to assess driving performance. Following the simulation, a second non-driving assessment on the n-back task was carried out. To control for differences in driving speed across 
each of the $3200 \mathrm{~m}$ assessment periods, each section of roadway was subdivided into $7.62 \mathrm{~m}$ (25ft) segments for analysis. Mean velocity and lane position were computed for each segment and then grand means and standard deviations determined. In the computation of the standard deviation of lane position, segments associated with lane changes were not included.

\section{RESULTS}

\section{Sample}

The sample consisted of 135 participants, 72 in the US and 63 in Korea. Gender was balanced by age and culture. US participants averaged 23.6 $(\mathrm{SD}=2.1)$ and $63.3(\mathrm{SD}=3.0)$ years for the two age groups. Koreans were nominally older $\mathrm{F}(1,131)=3.33, \mathrm{p}=.07$, averaging $24.6(\mathrm{SD}=2.3)$ and $63.9(\mathrm{SD}=2.7)$ years. Korean participants reported on average poorer physical well-being than US participants, $\mathrm{F}(1,131)=54.79, \mathrm{p}<.001$. Culture and age were not directly related to driving frequency but interacted, $\mathrm{F}(1,130)=5.97, \mathrm{p}=.016$. Older US participants drove more frequently than younger US participants while in Korea the inverse was true.

\section{Cognitive Task Performance}

Cognitive task performance was assessed as an error rate computed as the percentage of incorrect or non-responses to the total number of stimuli. Error rates are reported in Table 1. As expected, errors increased under dual-task conditions, $\mathrm{F}(1,130)=19.93, \mathrm{p}<.001$. Older participants committed an error $18.4 \%$ more often than the younger group $(F(1,130)=67.76, p<.001)$. US participants out performed Korean participants by scoring correctly $7.4 \%$ more often $(\mathrm{F}(1,130)$ $=11.01, \mathrm{p}=.001)$. A significant age $*$ culture interaction, $\mathrm{F}(1,130)=8.60, \mathrm{p}=.004$, indicates that while scores for younger participants across the two cultures do not differ significantly, the older Korean participants had more difficulty with the n-back task than older US participants. There was no interaction between the repeated measure and other independent variables, suggesting that differences in n-back performance by age and culture did not vary with total workload.

Table 1. Composite error scores on the secondary task (as percentages)

\begin{tabular}{llll}
\hline & & Non-driving & Dual-task \\
\hline US & Younger & $2.39(3.10)$ & $7.91(9.20)$ \\
Drivers & Older & $14.83(13.94)$ & $19.19(12.79)$ \\
Korean & Younger & $4.43(6.77)$ & $7.60(9.98)$ \\
Drivers & Older & $28.81(24.09)$ & $33.18(21.64)$ \\
\hline
\end{tabular}

Note: Table entries are mean percentages with the standard deviation in parentheses.

\section{Driving Performance}

Forward velocity. Older participants drove slower on the simulated highway than did younger drivers by an average of $1.6 \mathrm{~m} / \mathrm{s}, \mathrm{F}(1,131)=15.4, \mathrm{p}<.001)$. As shown in Figure 2, this pattern was consistent across cultures, with each older driver sample showing a lower mean velocity than their cultural cohort (no significant interaction of age by culture, $F(1,131)=.74, p=0.390$ ). Comparing cultures on driving speed, the US participants drove faster than the Koreans by $1.9 \mathrm{~m} / \mathrm{s}, \mathrm{F}(1,131)=22.86, \mathrm{p}<.001$. 
As expected, there was a main effect of workload on forward velocity, $F(2,262)=44.97$, $\mathrm{p}<.001$. Drivers decreased their speed an average $1.33 \mathrm{~m} / \mathrm{s}$ while engaged in the cognitive activity and increased speed again afterward by $1.29 \mathrm{~m} / \mathrm{s}$ (both pairwise comparisons significant, $\mathrm{p}<.001)$. Both age and culture interact with workload, $\mathrm{F}(2,262)=3.39, \mathrm{p}=.035$, and $\mathrm{F}(2,262)$ $=6.54, p=.002$ respectively. An inspection of the means shows that, as a group, older drivers showed a somewhat greater relative decrease in speed during the dual task. In addition, the Korean sample showed the most pronounced slowing, particularly in the older drivers.

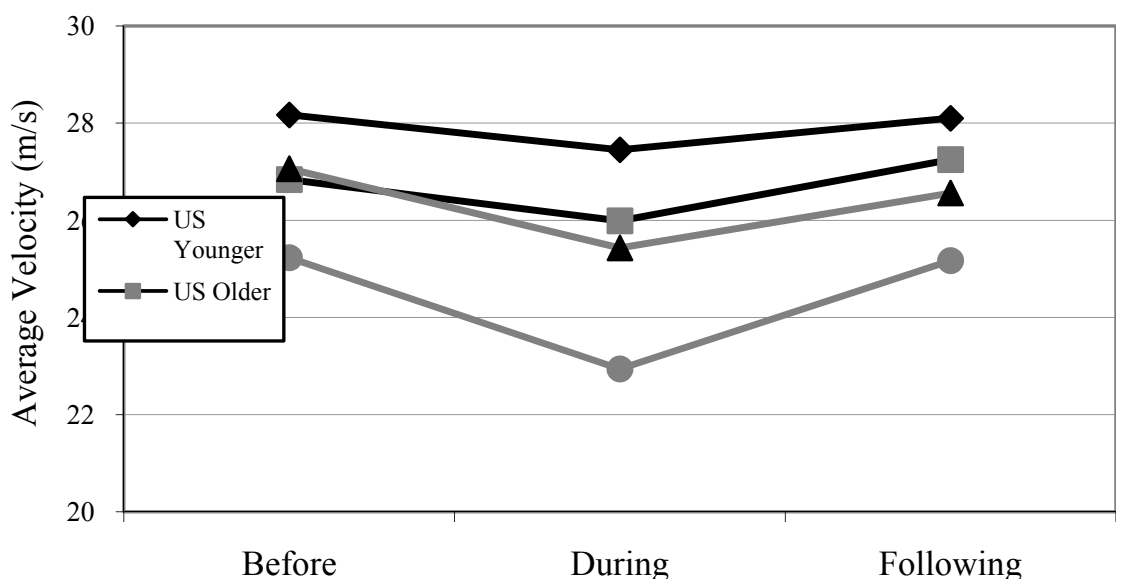

Figure 2. Forward velocity by age and culture

Speed control. Variability of speed control was higher in older drivers, $F(1,131)=13.45$, $\mathrm{p}<.001$. This is expressed in Figure 3 as the percent coefficient of variation of velocity which takes into account the base speed of the driver. There was also an effect of culture $(\mathrm{F}(1,131)=$ $50.23, \mathrm{p}<.001)$ with the Korean drivers showing greater variability in speed control.

There was a main effect of cognitive load on speed control, $F(1.82,238.30)=4.05, p=.022$ (Greenhouse-Geisser correction for sphericity). While planned comparisons show that the variability of speed control increased for the group as a whole during secondary task $(p=.002)$, it is clear that this effect was most pronounced in the older drivers. This appears as an age by cognitive load interaction, $\mathrm{F}(1.82,238.30)=3.77, \mathrm{p}=.028$. The younger Korean sample shows a somewhat divergent pattern in that variability in speed control did not decrease following the secondary task like the two older samples or remain constant as in the younger US drivers.

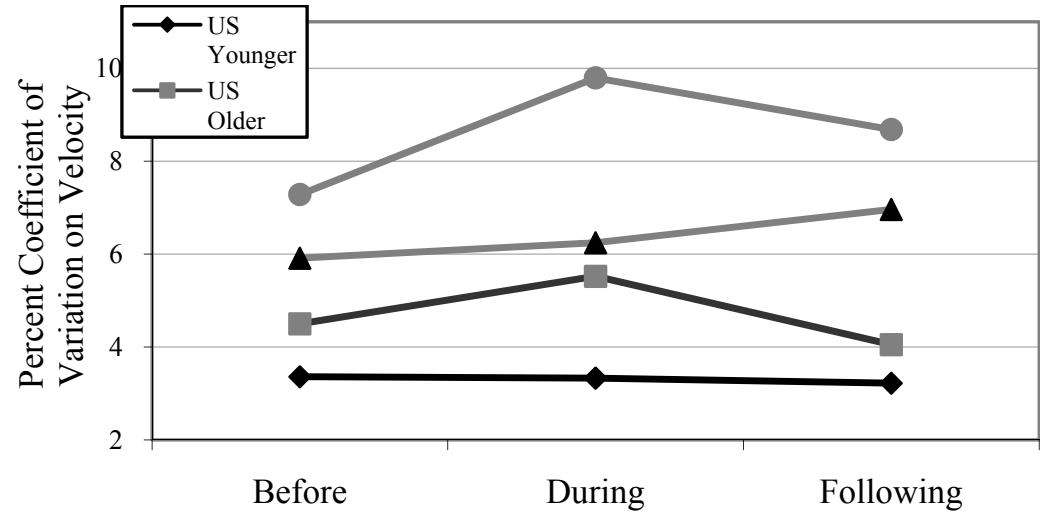

Figure 3. Percent coefficient of variation on velocity by age and culture 
Lateral control. Lateral control expressed as the standard deviation of lane position is shown in Figure 4. A main effect for age $(\mathrm{F}(1,130)=5.17, \mathrm{p}=.025)$ indicates that older participants had more difficulty maintaining lane discipline. Similarly, a main effect of culture on lateral control $(\mathrm{F}(1,130)=5.18, \mathrm{p}=.025)$ reflects greater variability in the Korean sample. Inspection of Figure 4 shows that these differences are driven to a large extent by the older Korean drivers. Consistent with an earlier simulation and field studies on younger participants using n-back tasks (Mehler et al., 2009; Reimer, 2009), drivers across the age and culture groups showed a significant reduction in lateral variation during the dual task, $\mathrm{F}(2,260)=17.80, \mathrm{p}<.001$.

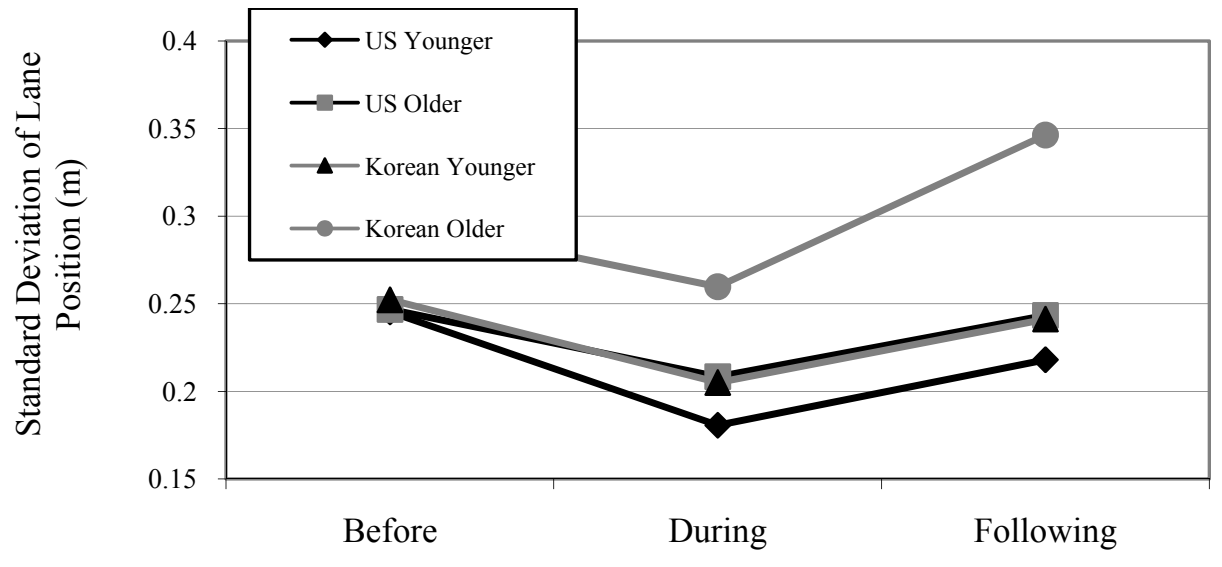

Figure 4. Standard deviation of lane position by age and culture

\section{DISCUSSION}

The age and culture subgroups showed a parallel decrement in performance on the n-back task during simulated driving relative to their performance under non-driving conditions. This may be taken as evidence that each group invested a comparable amount of their available cognitive resources in the n-back task during driving relative to their overall capability to perform the task under single task conditions. The introduction of the cognitive task during driving resulted in a compensatory slowing of forward velocity followed by an increase in speed when workload was reduced, extending previous findings (Mehler et al., 2008; Reimer, Mehler et al., 2006). This compensatory pattern was present across age and culture. The coefficient of velocity, an index of speed control, was negatively impacted by the dual task demands in the older driver samples. Each of the age and culture groups showed a decrease in the standard deviation of lane position during the added load of the secondary task. This pattern is likely due to a rigidification of control as attention is divided between the tasks (Reimer, 2009). The impact of age on all of the driving performance measures was most striking in the older Koran sample. Given their notably lower baseline n-back scores and poorer self-reported health status, it is possible that individuals recruited for the older Korean sample were functionally, if not chronologically, older than the US sample. As a cultural comparison, US drivers tended to drive the simulator somewhat faster than their Korean counterparts but with more consistent speed control. However, the overall patterns of change in driving performance in response to increased cognitive load were quite consistent across cultures in this highway simulation. The only obvious exception was the younger Koreans' increase in variability of lane position following the secondary task. We are currently 
evaluating the urban portion of simulation to assess whether driving context influences these relationships.

\section{ACKNOWLEDGMENTS}

The authors would like to thank Alex Pina, Kamo Jurn, Sung Kim, Alea Mehler, Shannon Roberts, Dorothy Brown, Kristin Malakorn, Rebecca Hung, Sandhya Ramakrishnam, Man Ho Kim, Yongtae Lee and Junhyung Bae for their assistance with data collection and the presentation of this research. This research was supported in part by the New England University Transportation Center at MIT and the Daegu Gyeongbuk Institute of Science and Technology (DGIST) Basic Research Program of the MEST.

\section{REFERENCES}

Boyle, J., Dienstfrey, S., \& Sothoron, A. (1996). National survey of speeding and other unsafe driving actions (No. DOT HS 808 749). Washington, DC: National Highway Traffic Safety Administration.

D'Ambrosio, L. A., Meyer, J., Coughlin, J. F., \& Mohyde, M. (2008). Self-regulation among older drivers: a cost-benefit approach. Unpublished manuscript, Cambridge, MA.

Folstein, M. F., Folstein, S. E., \& McHugh, P. R. (1975). Mini Mental State: A practical method for grading the cognitive state for the clinician. Journal of Psychiatry Research, 12(3), 189198.

Harms, L. (1991). Variation in drivers' cognitive load: Effects of driving through village areas and rural junctions. Ergonomics, 34(2), 151-160.

Matthews, G., Tsuda, A., Xin, G., \& Ozeki, Y. (1999). Individual differences in driver stress vulnerability in a Japanese sample. Ergonomics, 42(3), 387-414.

McDowd, J. M., Vercruyssen, M., \& Birren, J. E. (1991). Aging, divided attention, and dual-task performance. In D. L. Damos (Ed.), Multiple-task performance (pp. 387-414). London: Taylor \& Francis Ltd.

Mehler, B., Reimer, B., Pohlmeyer, A.E. \& Coughlin, J. F. (2008). The association between heart rate reactivity and driving performance under dual task demands in late middle age drivers. Advances in Transportation Studies, An International Journal, Special Issue.

Mehler, B., Reimer, B., Coughlin, J. F., \& Dusek, J. A. (2009). The impact of incremental increases in cognitive workload on physiological arousal and performance in young adult drivers. Paper presented at the Transportation Research Board annual meeting, Washington, DC.

Reimer, B. (2009). Cognitive Task Complexity and the Impact on Drivers' Visual Tunneling. Paper presented at the Transportation Research Board annual meeting, Washington, DC.

Reimer, B., D'Ambrosio, L. A., Coughlin, J. F., Kafrissen, M. E., \& Biederman, J. (2006). Using self-reported data to assess the validity of driving simulation data. Behavior Research Methods, 38(2), 314-324. 
Reimer, B., Mehler, B. L., Pohlmeyer, A. E., Coughlin, J. F., \& Dusek, J. A. (2006). The use of heart rate in a driving simulator as an indicator of age-related differences in driver workload. Advances in Transportation Studies an International Journal, Special Issue, 9-20.

Rogers, W. A., \& Fisk, A. D. (2001). Understanding the role of attention in cognitive aging research. In J. E. Birren \& K. W. Schaie (Eds.), Handbook of the psychology of aging. Oxford, UK: Elsevier Science.

Stutts, J. C., \& Hunter, W. W. (2003). Driver inattention, driver distraction and traffic crashes. ITE Journal, 73(7), 34-45.

Zeitlin, L. R. (1993). Subsidiary task measures of driver mental workload: A long-term field study. Transportation Research Record 1403, 23-27. 